\title{
Optimization of a multi-touch sensing device for biomedical applications
}

\author{
Septimiu Crisan ${ }^{1,}$, , Ioan Gavril Tarnovan ${ }^{1, b}$ \\ ${ }^{1}$ Department of Electrical Engineering and Measurements, Faculty of Electrical Engineering, \\ Technical University of Cluj-Napoca, Str. Memorandumului nr. 28, 400114, Cluj-Napoca, Romania \\ aseptimiu.crisan@ethm.utcluj.ro, bioan.tarnovan@ethm.utcluj.ro(corresponding author)
}

Keywords: Biomedical devices, Optical touch, Multi-touch systems.

\begin{abstract}
Multi-touch systems are redefining the natural user interface paradigm and their applications can be found ranging from mobile phones, tablets and screens to the control of industrial facilities. While the concepts of multiple touch sensing are not new, there are still large unexplored areas regarding optimization of the user experience for various industrial or medical applications. Along with the ability to detect and process simultaneous touches and gestures, large scale multi-touch devices offer collaborative work along with user-selective content management systems - features rarely used in dedicated medical visualization or sensing multi-touch software applications.

Based on previous research concerning multi-touch systems and their potential usefulness in the medical field, this paper describes the optimization process of a multi-touch sensing device for biomedical applications. Three important layers of a multi-touch device were chosen as candidates for optimization: sensing, data manipulation and visualization. The results were applied to a prototype optical touch system developed for multi-user/multi-touch environments and several hardware and software modifications were designed and implemented. Since the goal of this research was to explore ways to enhance the user experience in multi-touch applications the conclusions derived from this paper can be extended to other domains where concurrent visualization and processing of information are vital components.
\end{abstract}

\section{Introduction}

Touch sensing systems have been used for decades as information visualization and control devices in a multitude of areas and domains. However, true integration in mainstream applications has only been possible recently with the advent of multi-touch devices that allow simultaneous touches and objects to be tracked and interpreted. Moreover, the concept of multi-touch brings an important feature, the ability to process entries from more than one user at a time, creating the basis for collaborative work. The form factor of these devices has varied from small phones and tablets to large-scale interactive tables and walls and while multi-user sensing is not feasible on small size screens, large devices can benefit from such an implementation.

There are many domains of interest where natural user interfaces can improve process control and information visualization by employing multiple user collaborative applications. As part of the authors' ongoing research, developing biomedical applications revealed a domain where -due to the nature of the rich data visualization requirements - multi-touch, multi-user systems can be successfully used for visual data manipulation. As mentioned in [1], 'Traditional computing workflows 'coagulate' our coordination behavior of supposedly collaborative tasks into a sequential nature' therefore the multi-user collaborative environment of a multi-touch device brings us closer to the natural user interface paradigm.

The paper will enumerate the most important methods and technologies used to create multitouch, multi-user devices, assess user interaction and user experience in medical data visualization and describe an optimization process of previously researched multi-touch systems for biomedical applications. 


\section{Multi-touch, multi-user technologies}

As mentioned in the introduction, mainstream integration of touch devices was performed after the successful implementation of simultaneous sensing of multiple touches. As described in recent studies [1,2] multi-touch technologies are reaching maturity in Europe and North America with an estimated market share of 5.5 billion USD by 2016.

Multi-touch methods and applications have been thoroughly presented in research reports and articles $[3,4,5,6]$. They can be classified into four main categories based on the physical phenomenon that is employed in the process:

- Resistive sensing

- Capacitive detection (projected and surface)

- Surface acoustic waves (SAW)

- Optical sensing (Frustrated Total Internal Reflection, Diffused Illumination, Laser Light Plane, Diffused Surface Illumination, Edge Detection etc.)

Resistive touch sensing is one of the oldest methods used in actual touch devices but it is mainly a single touch technology with a few exceptions [7]. It is a technology that allows for excellent touch detection accuracy in comparison with other methods but requires more touch pressure. In addition, due to its low price, it is one of the most used methods for single touch information/visualization screens.

Discovered earlier than resistive detection, capacitive sensing has now reached a maturity stage that makes it an excellent choice for small-scale multi-touch enabled phones and tablets. Projected capacity has lower accuracy than resistive sensing but allows multiple point detection and it is more resilient due to the possibility to mount a rigid transparent layer over the capacitive conductors that form the detection grid. One of the main disadvantages is its inability to easily scale to higher dimensions due to the prohibitive manufacturing costs and collaborative devices built with this technology are not usually accessible to the general public.

Surface acoustic wave (SAW) touch screens track point interactions by measuring the attenuation of acoustic waves across the surface of a medium such as glass. The response is less precise than capacitive touch screens and surface acoustic wave technology's touch functions are affected by contaminants on the surface substrate [1].

Optical methods used to detect touch points fall under many categories depending on the placement of sensors and illumination modules. One common trait of these devices is the possibility to create affordable large-scale systems with true multi-touch capabilities. The enlarged physical dimensions represent the downside of such a system with the exception of some proprietary optical technologies capable of low depths, as is the case with Microsoft Pixelsense [8].

Multi-touch methods comparison. A side by side comparison of parameters regarding user interaction and experience is presented in a research performed in [9] and is depicted in figure 1.

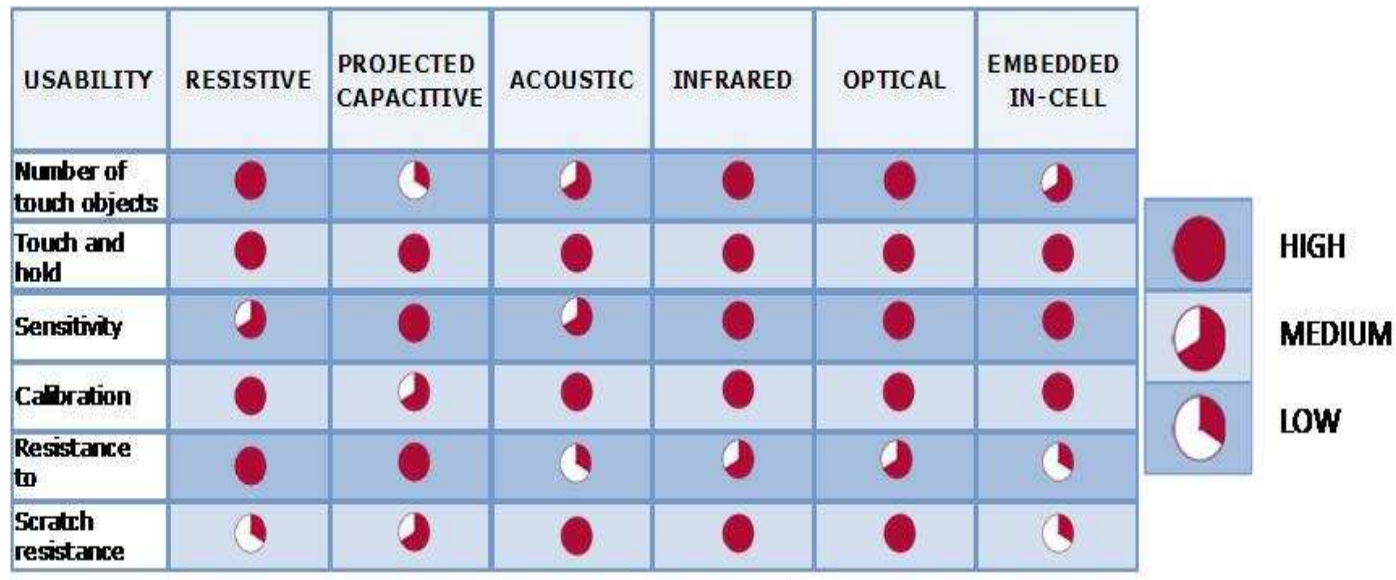

Fig. 1 Usability assessment of major touch technologies as described in [9] 
As technology advances, it is likely that the characteristics of all methods will become similar in the recent future and general usability will depend on ergonomic criteria rather than the technology employed.

Figure 2 shows the market share for touch technologies in 2010 according to [2]. It is important to note that the chart assesses both single touch and multi-touch technologies and therefore resistive sensing is used in 50\% of the existing devices while not allowing multi-touch. As described in [1], there are three main classes of multi-touch devices based on their dimensions and screen diagonal mobile devices (palm and tablet sizes, $7.5 \ldots 25 \mathrm{~cm})$, desktop monitors $(38 \ldots 75 \mathrm{~cm})$, and collaborative multi-touch devices (above $75 \mathrm{~cm}$ ). The representation in figure 2 takes into consideration all form factors thus accounting for smartphones and tablets, which greatly surpass large-scale devices in number of units sold.

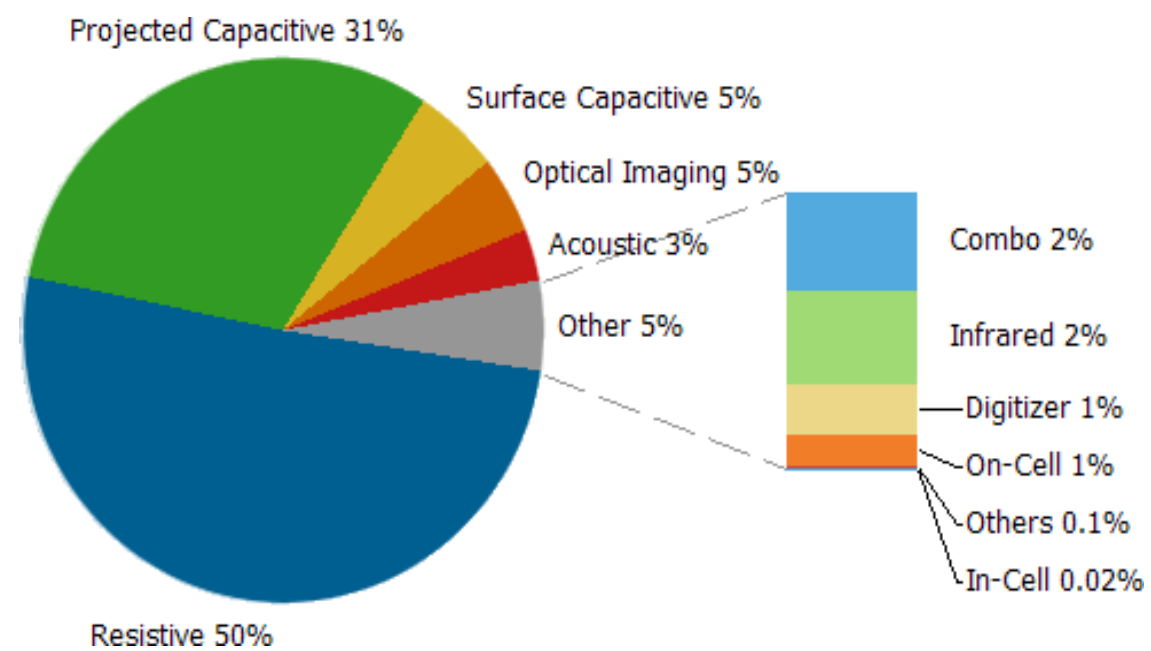

Fig. 2 Market share for touch technologies in 2010 [2]

While the initial study for this research has not uncovered a description of the market share for large multi-touch, multi-user devices, based on the major players in the market it is probably a standoff between thin optical sensing and projected capacitive $[8,10]$.

As an important part of the authors' research, optical touch screens allow for fast prototyping and integration in various domains and they are mostly suited for tabletop interaction due to their large dimensions. This ability permits an early assessment of the user interaction with a multi-touch device in various domains and the user experience can be quantified by analyzing user behavior and interaction patterns.

As a crucial domain, medical devices and applications can benefit from the added value brought by the touch capable, multi-user collaborative environment.

Multi-touch interaction for medical data. Due to the nature of the medical field, information regarding patient status is often visual and comes from a variety of sources. Even more, the sources contain proprietary information that can be displayed only on the designated device. The DICOM standard has managed to solve some of these incompatibilities, clinical information being available under a common standard ready for visualization. This in turn creates a perfect premise for the introduction of multi-touch screens as real time data manipulation devices. While the integration of the DICOM model in a multi-touch application is a trivial task, the collaborative work requires the development of a context and user based hierarchical system.

For a centralized system containing relevant patient data, several experiments were performed using a previously built general-purpose multi-touch multi-user system named "Vision Deck" [11, 12].

Figure 3 shows data manipulation for a patient X-ray on the Vision Deck platform. 


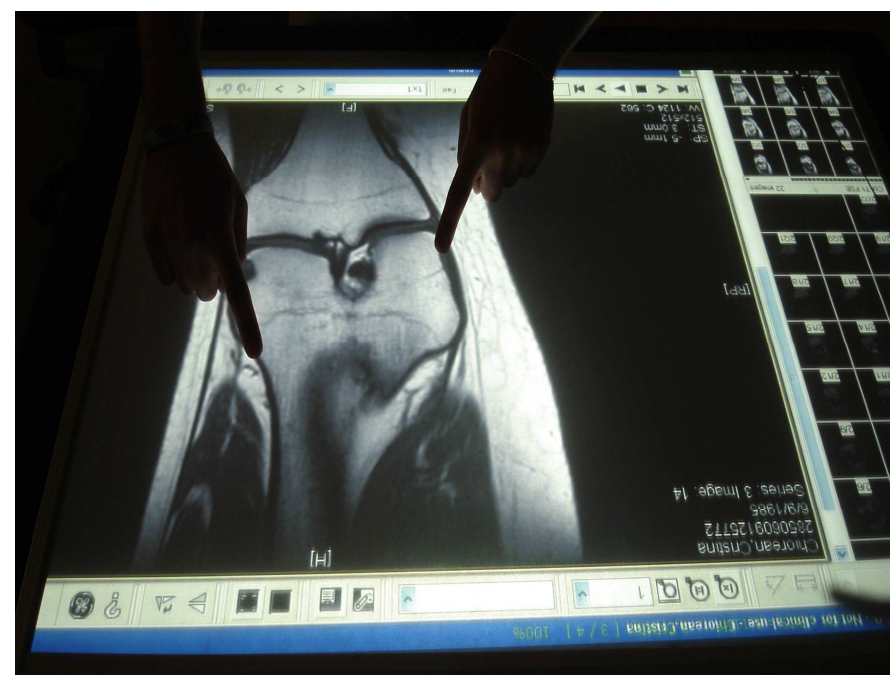

Fig. 3 Visual data manipulation on the Vision Deck system

Experiments involved data visualization and manipulation, streamlined for several physicians according to their specialty. The application built specifically for the experiments involved an administrative module for non-medical patient data, a multi-touch, multi-user medical visualization module built on top of the DICOM standard and a hierarchical content management system[12].

Several problems were encountered mainly due to unsatisfactory user experience and interaction. While the hardware system accurately detected touches and gestures, the FTIR optical technology involved the use of an external compliant layer that -while allowing doctors wearing gloves to interact with the system- accumulated dirt and developed wrinkles due to increased friction and prolonged use. Along with the hygienic problems, the required pressure for swipe gestures and 'drag and drop' operations added an uncomfortable sensation reported by the users of the device. The sensing module underwent important modifications and for the medical applications, a completely different technology has been employed. By replacing the top part of Vision Deck with a module using the Laser Light Plane (LLP) method, the need for a soft compliant layer has been eliminated. LLP can detect hovering touches at $1 \ldots 3 \mathrm{~mm}$ above the surface rendering obsolete the need to apply pressure on the device. In addition, this change resulted in the top layer of the device being made out of reinforced acrylic that can be easily cleaned and has $40 \%$ less friction between the users' fingers and the material. As LLP does not support passive objects (fiducials) on the table, Vision Deck has been provided with six lasers instead of the minimum recommended four in order to prevent occultation when using self-illuminated fiducials.

Another reported problem dealt with the content management system. The algorithm for granting access to data manipulation for different medical procedures and scans has been thought of having a strong connection with the ID of the physician and as a result with its own specialty. While the approach works in an industrial setup where hierarchy and specialization define the organizational chart, for a medical management application it falls short of its objectives. Medical personnel, while highly specialized, are capable of strong knowledge connections with adjacent domains, i.e. an orthopedic surgeon is able to read and interpret X-rays, most doctors are familiar with general medicine etc. The content presentation has been streamlined so that physicians get access to all relevant data but the data closely related to their primary occupation is displayed first. In addition, the system now offers the possibility to add annotations and has a general access log available to the management level.

For the actual visualization layer, optimization has been applied to popup context menus that had previously created with a fixed orientation thus hindering the collaborative work. When more than one user accesses the table, content has to be delivered according to the individual user orientation. For any visual patient data on display, the annotations tab and the context menus now mimic the medical image orientation insuring correct information delivery. 
Along with visualization touch devices, optical multi-touch systems designed to be used as biomedical devices can be constructed to serve as diagnose assistants for a precise set of measurements - as is the case with optical podoscopes. Based on the lessons learned while constructing the Vision Deck multi-touch system, an optical podoscope was built in [13] as a biomedical tool to serve in the diagnosis of static and dynamic foot unbalance scenarios. Since the podoscope uses similar optical touch sensing technology as a general purpose multi-touch table, it was chosen as a proof of knowledge for optimizing the sensing module in a real biomedical application comprising both hardware and software requirements. In addition, optical podoscopes are an emerging niche in orthopedics' science where a touch-sensing device can offer additional information as long as they are optimized enough in comparison with classical devices.

\section{Optimization of an optical touch podoscope}

The optical podoscope uses a multiple lighting setup by employing both the FTIR - Frustrated Total Internal Reflection technique and the DI -Diffused Illumination method. A representation of the original device can be observed in figure 4

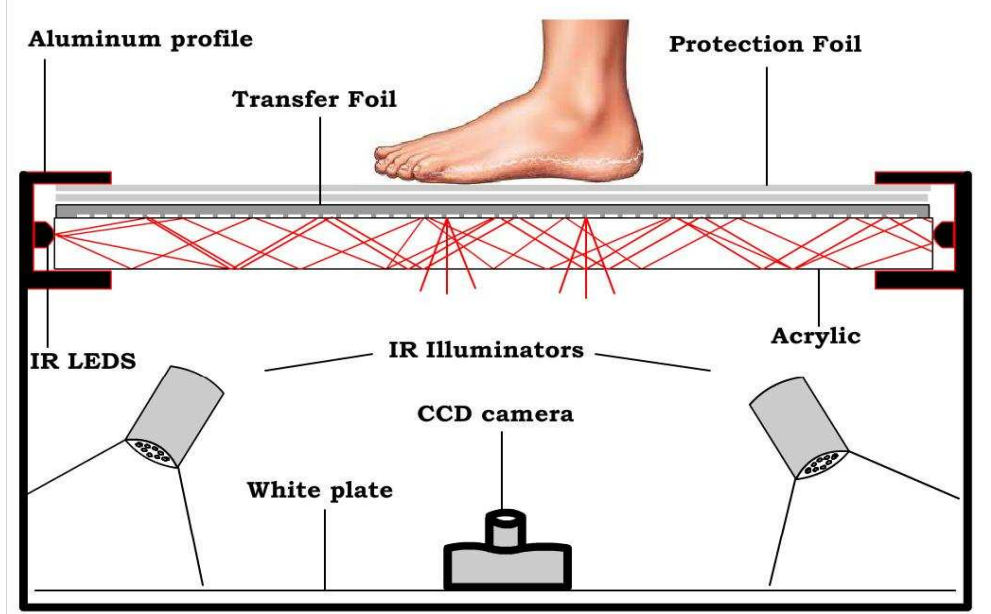

Fig. 4 Schematic of the original computer assisted optical podoscope built in [13]

While the original device served its purpose as a tool to assist in the assessment of foot disorders, both the sensing module and the data manipulation/visualization routines were at a prototype level lacking advanced features that could add significant value to the interaction between orthopedist, patient and sensing device.

The first step of the optimization process dealt with the general "anatomy" of the device, more specifically, trying to reduce the depth of the device while keeping the sensing area as large as needed for gathering patient data. This is a common problem for most optical multi-touch devices that have to rely on a video camera for acquisition because of the long optical path required. Even more, in normal setups, the presence of a video projector as the image source introduces the necessity for even larger depths due to the image throw distance. In the same vein, reducing the physical dimensions of the optical touch podoscope is of great importance because it allows patients to position themselves on the testing area easier and eliminates the need for an additional tread or riser.

Since the dimensions are calculated based on the optical characteristics of the CCD Camera responsible for acquiring images, several experiments were performed using wide-angle lenses. Various mounts and lenses were chosen starting from the $1.9 \mathrm{~mm} 109$ degrees field of view (FoV) M12 mount lens up to the $4.3 \mathrm{~mm} \mathrm{m12}$ Lens with 70 degrees field of view (FoV). The tests undertaken dealt with measuring the distorition/barrel effect added by extreme wide angle lens. While the largest FoV is desired for reducing the depth of the device, for practical reasons a 79 degrees FoV lens has been chosen since correcting the optical aberations becomes a burden on the image processing pipeline at more than 85 degrees FoV. 
A snapshot of some of the images acquired with the detection camera and taken from the experiments is depicted in figure 5. All images were scaled or cropped to display the usable sensing area even if in the case of wide „fisheye“ lenses the resulting image contained parts of the device which do not belong to the region of interest.

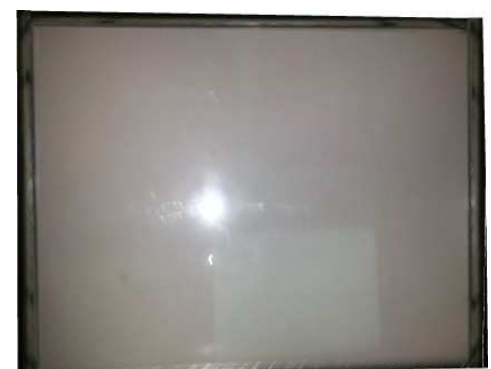

a) $3.6 \mathrm{~mm} 79$ degrees FOV

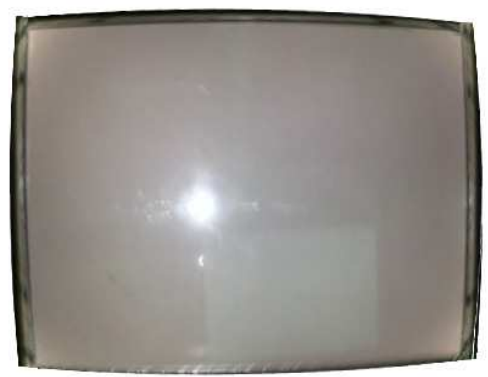

c) $2.1 \mathrm{~mm} 102$ degrees FOV

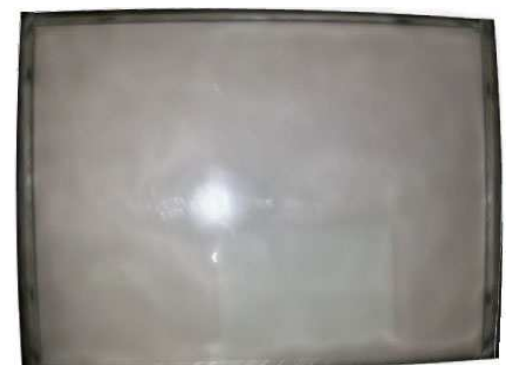

b) $2.8 \mathrm{~mm} 92$ degrees FOV

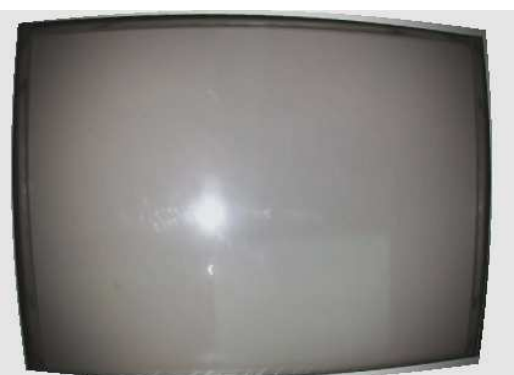

d) $1.9 \mathrm{~mm} 106$ degrees FoV

Fig. 5 Barrel distortion lens experiment on the optical touch podoscope. Image of the podoscope sensing screen

In order to solve the barrel effect problem, a software module has been devised that corrects the optical effect by warping the image with a reverse distortion as the parameters of the lens are known. The latency introduced by the processing filter adds up to $8 \mathrm{~ms}$ for each frame - an acceptable tradeoff even for gait tests.

In addition to the introduction of wide angle lenses, the optical path of the video camera has been folded by using a first surface mirror placed on the bottom of the optical podoscope as seen in figure 6 .

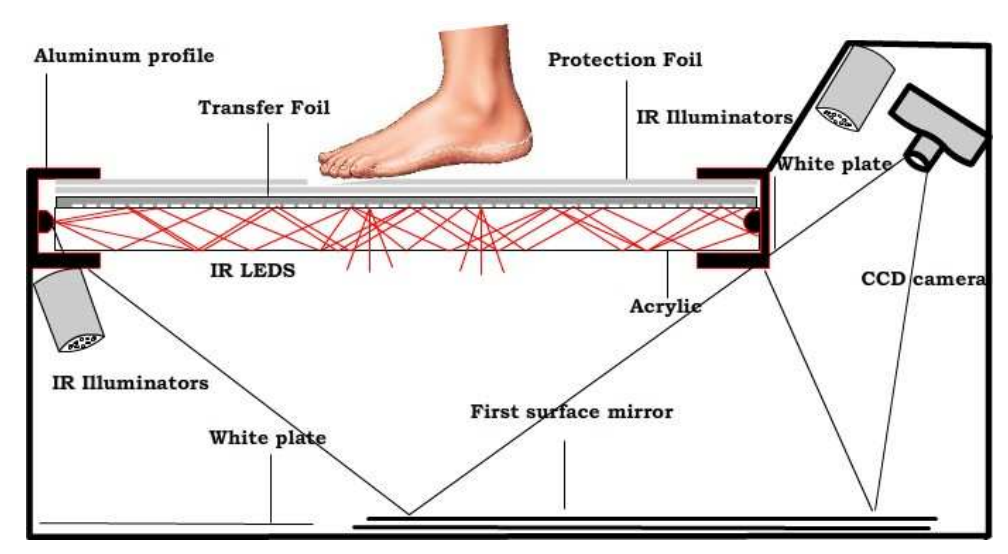

Fig. 6 Optical path folding and new lighting configuration for the optimized podoscope

Two experiments were performed using normal glass and acrylic mirrors that have a considerably lower acquisition price than first surface mirrors. The experiments have shown that in the case of analyzing the interaction between the sole and the hard acrylic surface, the ghosting 
phenomena led to the creation of visual artifacts. Discrete changes in the foot pressure can lead to a better diagnosis and the two layers of normal mirrors fused the acquired intensity of neighboring pixels in the image. A description of the ghosting effect is seen in figure 7

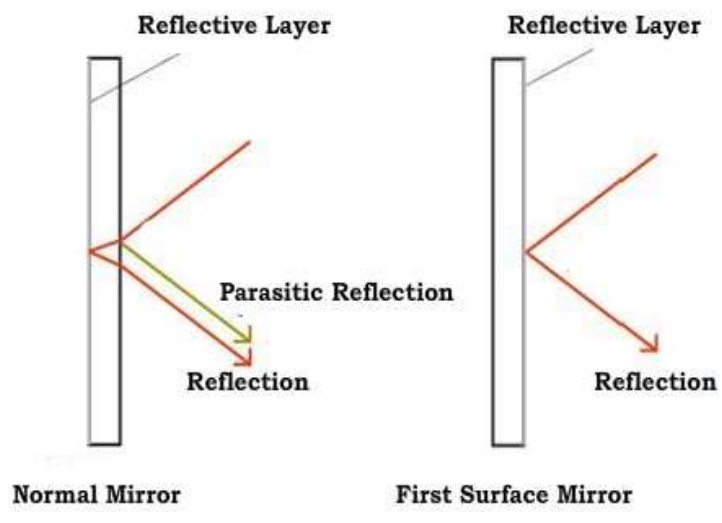

Fig. 7, Ghosting phenomena due to double layers (reflective and glass) in normal mirrors

Modifications were also performed on the processing pipeline and presentation layer. While being a multi-touch device, the podoscope does not possess an embedded screen to display image data. Instead, data is processed and shown on an outside monitor connected to the processing computer, thus eliminating the need for image calibration, low friction foils or fiducial detection. This offers superior processing speed than a normal multi-touch device -unnecessary for static analysis but vital for dynamic assessments. With the added optical distortion compensation algorithm, in order to maintain a speed of 60 frames processed per second, the contour detection algorithm now determines the region of interest for the acquisition and image corrections are performed only on the new ROI. All image presentation and enhancement options are reserved for the post processing stage.

In a complex usage scenario with a region of interest totaling $40 \%$ of the complete image area, the processing pipeline has a latency of $14 \mathrm{~ms}$ representing an increase from the desired 60 frames per second.

\section{Conclusions}

Medical visualization and detection applications have important traits that require a particular approach in designing both hardware and software solutions for multi-touch collaborative devices. While implementing touch devices for the medical field has been a research goal of the authors, several problems appeared when the step from simulation to real interaction has been taken. Medical integration of multi-touch, multi-user devices is a new topic in scientific literature and this prevents performance comparisons to other devices, ipsative assessments representing the current evaluation technique. This paper analyzes some of the challenges encountered and offers streamlined solutions for optimizing sensing, presentation and manipulation layers for previously built prototype multi-touch multi-user systems. The hardware and software modifications have been outlined in the paper and in the case of a touch sensitive optical podoscope the proposed changes have been thoroughly detailed.

The results gathered are just a first step in designing robust touch sensing devices that can become a viable solution to the distributed information problem in the medical field. The ultimate goal is to deliver an industry-like command and control interface that allows unified access to relevant patient data in order to oversee and improve the healing process. 


\section{Acknowledgment}

This paper was supported by the project "Development and support of multidisciplinary postdoctoral programmes in major technical areas of national strategy of Research - Development Innovation" 4D-POSTDOC, contract no. POSDRU/89/1.5/S/52603, project co-funded by the European Social Fund through Sectoral Operational Programme Human Resources Development 2007-2013.

\section{References}

[1] Eugene Ch'ng, The Mirror Between Two Worlds: Multitouch-Multiuser Interaction for 3D Digital Objects and Environments, in Harrison, D. (ed.), Digital Media and Technologies for Virtual Artistic Spaces, IGI Global, (2013)

[2] Global Multi Touch Market, by Product (SmartPhones, Tablets, Laptops, Televisions/LCD, Tables, Floors), Applications (Entertainment, Infotainment, Enterprises, others) \& Geography, 2011-2016. Dallas, Texas: Marketsandmarkets.com.

[3] Schöning, J., Hook, J., Bartindale, J., Schmidt, D., Olivier, P., Echtler, F., et al., Building Interactive Multi-touch Surfaces. In C. Muller-Tomfelde (Ed.), TABLETOPS HORIZONTAL INTERACTIVE DISPLAYS (Vol. Technical Report (TUM-I0833)), Germany: Institute for Geoinformatics, University of Münster,(2010), pp. 27-49

[4] Hodges, S., Izadi, S., Butler, A., Rrustemi, A., \& Buxton, B.. ThinSight: versatile multi-touch sensing for thin form-factor displays, UIST '07 Proceedings of the 20th annual ACM symposium on User interface software and technology (2007)

[5] Çetin, G., Bedi, R., Sandler, S. (Eds.) Multi-touch Technologies. 1st edition http://www.nuigroup.com (2009)

[6] Jefferson Y. Han, Low-cost multi-touch sensing through frustrated total internal reflection, Proceedings of the 18th annual ACM symposium on User interface software and technology, Seattle, WA, USA, (2005)

[7] Stantum and Nissha Printing Unveil FineTouch Z, Reuters US, retrieved on 18.12.2012, http://www.reuters.com/article/2012/06/04/idUS30918+04-Jun-2012+BW20120604

[8] Steven Musil, Microsoft renames its old Surface computer as PixelSense, CNET news, retrieved on 18.12.2012, http://news.cnet.com/8301-10805_3-57455731-75/microsoftrenames-its-old-surface-computer-as-pixelsense/

[9] DisplaySearch, Touch Panel Market Analysis (2010), http://www.displaysearch.com/cps/rde/ xchg/displaysearch/hs.xsl/100519_touch_screen_shipments_pass_600_million_in_2009.asp

[10] Ray Willington, Perceptive Pixel Outs 82" 1080p Multi-Touch Display, retrieved on 19.12.2012, http://hothardware.com/News/Perceptive-Pixel-Outs-82-1080p-MultiTouchDisplay/

[11] S. Crisan, V. D. , Zaharia, L. V. Brender, T.E. Crisan, A multi-touch collaborative solution for measurement data visualisation, XIX IMEKO World Congress, Lisbon Portugal (2009)

[12] S. Crisan, I. G. Tarnovan, T. E. Crisan, B. Tebrean, Optical Multi-touch System for Patient Monitoring and Medical Data Analysis, ADVANCEMENTS OF MEDICINE AND HEALTH CARE THROUGH TECHNOLOGY, IFMBE Proceedings, vol. 26, Springer (2009) 279-282

[13] S. Crisan, V. D. Zaharia, C. Curta and E. D. Irimia, Computer Assisted Optical Podoscope for Orthostatic Measurements, ADVANCEMENTS OF MEDICINE AND HEALTH CARE THROUGH TECHNOLOGY, IFMBE Proceedings, Volume 36, Part 3 (2011) 226-229 